\title{
Spontaneous Breakdown of the Definiteness in Some Convective Heat Transfer Problems
}

\author{
Eugen Magyari
}

Received: 22 October 2011 / Accepted: 16 November 2011 / Published online: 29 November 2011

(C) Springer Science+Business Media B.V. 2011

\begin{abstract}
In this study, it is shown that above a critical value of a governing parameter, the solutions of some convective heat transfer problems can undergo a bifurcation into a continuum of a non-denumerable infinity of solutions. Thus, the corresponding Nusselt number becomes indeterminate. The origin of this anomalous bifurcation resides in the stability change of the asymptotic state $\theta(\infty)$ from that of an unstable to that of a stable equilibrium point of the system. As a consequence, the boundary condition $\theta(\infty)=0$ becomes automatically satisfied and thus ineffective in determining the integration constants. Accordingly, the well-posed problem changes spontaneously into an ill-posed one. This remarkable phenomenon will be discussed in detail in the case of an unsteady forced and mixed convection heat transfer problem encountered in an article published recently in Transport in Porous Media. Subsequently, the mentioned loss of definiteness will be explained intuitively with the aid of a simple point-mechanical analogy.
\end{abstract}

Keywords Convective heat transfer - Anomalous bifurcation · Nusselt number . Unstable equilibrium $\cdot$ Stable equilibrium $\cdot$ Point mechanical analogy

\section{Introduction and Problem Formulation}

The aim of the present article is to explain the phenomenon already emphasized in the title and outlined shortly in the Abstract. Actually, the mentioned anomalous bifurcation of a well-defined solution into a continuum of a non-denumerable infinity of solutions can be observed in several convection boundary value problems both in porous media and in clear viscous fluids. To be specific, this phenomenon will be explained here with reference to an unsteady forced and mixed convection boundary value problem encountered in an article of Rohni et al. (2011) published recently in Transport in Porous Media.

E. Magyari (ه)

Departement Physik, Universität Basel, Klingelbergstr. 82, 4056 Basel, Switzerland

e-mail: magyari@bluewin.ch 
In the article of Rohni et al. (2011), the unsteady mixed convection boundary layer flow with suction and temperature slip near the stagnation point on a vertical surface embedded in a saturated porous medium has been investigated numerically and analytically. It has been assumed that the velocity of the driving external stream involves the classical "hyperbolic time variation"

$$
u_{\mathrm{e}}(t, x)=\frac{a_{0} x}{1-\gamma t}
$$

which has first been considered by Yang (1958) in a clear-fluid counterpart of the problem examined by Rohni et al. (2011). In addition, in Rohni et al. (2011), the effects of thermal buoyancy and of temperature slip have been taken into account, assuming that the relative wall temperature distribution $T_{\mathrm{w}}(t, x)-T_{\infty}$ without temperature slip is proportional to $u_{\mathrm{e}}(t, x)$. Then, following Mukhopadhyay and Andersson (2009), the time dependence of the temperature slip factor has been chosen in such a way that a similarity reduction of the problem becomes possible. Furthermore, using for the velocity the similarity transformation of Yang (1958) and for the temperature field the classical Pohlhausen ansatz $T=T_{\infty}+\left(T_{\mathrm{w}}-T_{\infty}\right) \theta$, the governing balance equations and the pertinent boundary conditions have been reduced for the similar stream function $f(\eta)$ and the similar temperature field $\theta(\eta)$ to the two-point boundary value problem (Rohni et al. 2011)

$$
\begin{gathered}
f^{\prime}=1+\lambda \theta, \\
\theta^{\prime \prime}+f \theta^{\prime}-f^{\prime} \theta-A\left(\theta+\frac{\eta}{2} \theta^{\prime}\right)=0, \\
f(0)=s, \quad \theta(0)=1+\Lambda \theta^{\prime}(0), \quad \theta(\infty)=0
\end{gathered}
$$

In the above equations the primes denote differentiations with respect to the similarity independent variable $\eta, \lambda$ is the mixed convection parameter, $\Lambda$ the temperature slip parameter, $s$ the mass transfer parameter, and $A=\gamma / a_{0}$ the unsteadiness parameter, as was defined in Rohni et al. (2011). (The condition $f^{\prime}(\infty)=1$ present in Eq. 11 of Rohni et al. (2011) has been omitted in our Eq. 2 since it is a direct consequence of the first Eq. 2 and of the asymptotic condition $\theta(\infty)=0$, and thus it is redundant). In order to avoid the occurrence of singularities at a finite time, in Rohni et al. (2011) $\gamma \leq 0$ has been assumed. This assumption restricts the variation range of the unsteadiness parameter $A=\gamma / a_{0}$ to $A \leq 0$, which corresponds to a decelerating flow for $A<0$ and to the steady flow for $A=0$. Hereafter, the assumption $A \leq 0$ will still be preserved and, correspondingly, $A$ will be replaced by $-|A|$.

In our present considerations, the unsteadiness parameter $A$ plays a key role and the other three parameters $(\lambda, \Lambda$ and $s$ ) could basically be set equal to zero, without affecting the main theme of the article. Specifically, in this respect, it will be shown that the parameters $\lambda, \Lambda$ and $s$ do not exert any influence on the critical value of $A$ where the mentioned anomalous bifurcation occurs.

\section{Forced Convection Solutions}

In the forced convection limit $\lambda=0$, the first Eq. 2 admits the elementary solution $f=\eta+s$ so that the boundary value problem (2) reduces to

$$
\begin{gathered}
\theta^{\prime \prime}+\left(\frac{2+|A|}{2} \eta+s\right) \theta^{\prime}-(1-|A|) \theta=0 \\
\theta(0)=1+\Lambda \theta^{\prime}(0), \quad \theta(\infty)=0
\end{gathered}
$$


These equations coincide with the respective Eqs. 17 and 11 of Rohni et al. (2011) where the forced convections considerations have been restricted to the impermeable case $s=0$. In the following, this latter restriction will be relaxed. In this case, the variable change

$$
z=-\frac{1}{4}\left(\sqrt{2+|A|} \eta+\frac{2 s}{\sqrt{2+|A|}}\right)^{2}
$$

transforms Eq. 3 in

$$
z \frac{\mathrm{d}^{2} \theta}{\mathrm{d} z^{2}}+\left(\frac{1}{2}-z\right) \frac{\mathrm{d} \theta}{\mathrm{d} z}-\frac{|A|-1}{|A|+2} \theta=0
$$

which is precisely Kummer's equation of the confluent hypergeometric function $M(a, b, z)$ (see Eq. 13.1.1, Abramowitz and Stegun 1965) with

$$
a=\frac{|A|-1}{|A|+2}, \quad b=\frac{1}{2}
$$

Thus, the general solution of Eq. 3 can be written as a linear combination of $M(a, b, z)$ and $z^{1-b} M(1+a-b, 2-b, \xi)$ (see Eqs. 13.1.12 and 13.1.13 of Abramowitz and Stegun $1965)$ in the form:

$$
\theta(\eta)=C_{1} M\left(a, \frac{1}{2}, z\right)+\frac{1}{2} C_{2}\left(\sqrt{2+|A|} \eta+\frac{2 s}{\sqrt{2+|A|}}\right) M\left(a+\frac{1}{2}, \frac{3}{2}, z\right)
$$

In this way, the wall temperature $\theta(0)$ and the wall temperature gradient $\theta^{\prime}(0)$ (i.e., the so-called reduced Nusselt number- $\left.-\theta^{\prime}(0)\right)$ are obtained in terms of $C_{1}$ and $C_{2}$ as

$$
\begin{aligned}
& \theta(0)=M\left(a, \frac{1}{2}, z_{0}\right) C_{1}+\frac{s}{\sqrt{2+|A|}} M\left(a+\frac{1}{2}, \frac{3}{2}, z_{0}\right) C_{2}, \\
& \theta^{\prime}(0)=-2 \text { as } M\left(a+1, \frac{3}{2}, z_{0}\right) C_{1} \\
&+ {\left[\frac{\sqrt{2+|A|}}{2} M\left(a+\frac{1}{2}, \frac{3}{2}, z_{0}\right)-\frac{(2 a+1) s^{2}}{3 \sqrt{2+|A|}} M\left(a+\frac{3}{2}, \frac{5}{2}, z_{0}\right)\right] C_{2} }
\end{aligned}
$$

where

$$
z_{0}=-\frac{s^{2}}{2+|A|}
$$

\section{Unique Forced Convection Solutions for $|A| \leq 1$ and Loss of Definiteness for $|A|>|A|_{\text {crit }}=1$}

The constants of integration $C_{1}$ and $C_{2}$ occurring in Eqs. 8-10 have to be determined with the aid of the boundary conditions (4). The uniqueness of solution (8) depends on whether the two Eq. 4 yield unique solutions for $C_{1}$ and $C_{2}$. In the following, we show that this is the case only for $|A| \leq 1$, while, above of the critical value $|A|_{\text {crit }}=1$, a spontaneous loss of definiteness occurs which persist in the whole range $|A|>1$ of the unsteadiness parameter. Bearing in mind that the new variable $z$ is negative, we obtain according to Eq. 13.1.5 of Abramowitz and Stegun (1965) the asymptotic behavior

$$
\theta(\eta) \underset{\eta \rightarrow \infty}{\sim} \sqrt{\pi}\left(\frac{2+|A|}{4}\right)^{-a}\left[\frac{C_{1}}{\Gamma\left(\frac{1}{2}-a\right)}+\frac{C_{2}}{2 \Gamma(1-a)}\right] \eta^{\frac{2(1-|A|)}{2+|A|}}
$$


where $\Gamma$ stands for the Euler Gamma function. In discussing Eq. 12, two cases must be distinguished, namely $|A| \leq 1$ and $|A|>1$, respectively.

3.1 The Range $|A| \leq 1$

In this range, in Eq. 12, the exponent of $\eta$ is positive or zero, and thus the asymptotic condition $\theta(\infty)=0$ can only be satisfied when

$$
\frac{C_{1}}{\Gamma\left(\frac{1}{2}-a\right)}+\frac{C_{2}}{2 \Gamma(1-a)}=0 \quad(|A| \leq 1)
$$

Now Eqs. 9 and 13 yield for the integration constants $C_{1}$ and $C_{2}$ the unique solutions in terms of $\theta(0)$

$$
\begin{gathered}
C_{1}=\frac{\Gamma\left(\frac{1}{2}-a\right) \theta(0)}{\Gamma\left(\frac{1}{2}-a\right) M\left(a, \frac{1}{2}, z_{0}\right)-\frac{2 s}{\sqrt{2+|A|}} \Gamma(1-a) M\left(a+\frac{1}{2}, \frac{3}{2}, z_{0}\right)}, \\
C_{2}=\frac{2 \Gamma(1-a) \theta(0)}{\Gamma\left(\frac{1}{2}-a\right) M\left(a, \frac{1}{2}, z_{0}\right)-\frac{2 s}{\sqrt{2+|A|}} \Gamma(1-a) M\left(a+\frac{1}{2}, \frac{3}{2}, z_{0}\right)}
\end{gathered}
$$

On the other hand, substituting Eq. 14 in Eq. 10 we get

$$
\theta^{\prime}(0)=-\Omega \theta(0)
$$

where

$\Omega=\frac{2 a s \Gamma\left(\frac{1}{2}-a\right) M\left(a+1, \frac{3}{2}, z_{0}\right)+\Gamma(1-a)\left[\sqrt{2+|A|} M\left(a+\frac{1}{2}, \frac{3}{2}, z_{0}\right)-\frac{2(2 a+1) s^{2}}{3 \sqrt{2+|s|}} M\left(a+\frac{3}{2}, \frac{5}{2}, z_{0}\right)\right]}{\Gamma\left(\frac{1}{2}-a\right) M\left(a, \frac{1}{2}, z_{0}\right)-\frac{2 s}{\sqrt{2+|A|}} \Gamma(1-a) M\left(a+\frac{1}{2}, \frac{3}{2}, z_{0}\right)}$

Accordingly, Eq. 15 and the first boundary condition (4) yield

$$
\theta(0)=\frac{1}{1+\Lambda \Omega}, \quad \theta^{\prime}(0)=-\frac{\Omega}{1+\Lambda \Omega} \quad(|A| \leq 1)
$$

Therefore, when $|A| \leq 1$, the solution is unique and is given by

$$
\theta(\eta)=\frac{\Gamma\left(\frac{1}{2}-a\right) M\left(a, \frac{1}{2}, z\right)-\left(\sqrt{2+|A| \eta}+\frac{2 s}{\sqrt{2+|A|}}\right) \Gamma(1-a) M\left(a+\frac{1}{2}, \frac{3}{2}, z\right)}{\Gamma\left(\frac{1}{2}-a\right) M\left(a, \frac{1}{2}, z_{0}\right)-\frac{2 s}{\sqrt{2+|A|}} \Gamma(1-a) M\left(a+\frac{1}{2}, \frac{3}{2}, z_{0}\right)} \theta(0)
$$

To be specific, in Table 1, the exact solution (18) and its derivative $\theta^{\prime}(\eta)$ have been given for $A=0$ and $A=-1$ in terms of the complementary error function erfc (.) explicitly.

It is also worth emphasizing here that the Kummer functions $M(a, b, z)$ occurring in the above equations have been defined on the negative half-axis $z$ (see Eqs. 5 and 11). However, with the aid of the Kummer transformation

$$
M(a, b,-|z|)=\mathrm{e}^{-|z|} M(b-a, b,|z|)
$$

(see Eq. 13.1.27 of Abramowitz and Stegun 1965) the $M$-functions can easily be mapped on the positive half axis $-z=|z|$, and thus the solution (18) can be expressed in terms of Kummer's $U$-function (defined by Eq. 13.1.3 of Abramowitz and Stegun 1965) as follows:

$$
\theta(\eta)=\mathrm{e}^{-\frac{2+|A|}{4} \eta^{2}-s \eta} \frac{U\left(\frac{1}{2}-a, \frac{1}{2},|z|\right)}{U\left(\frac{1}{2}-a, \frac{1}{2},\left|z_{0}\right|\right)} \theta(0)
$$


Table 1 Exact solutions of the forced convection boundary value problem (3), (4) for the values 0 and -1 of the unsteadiness parameter $A$

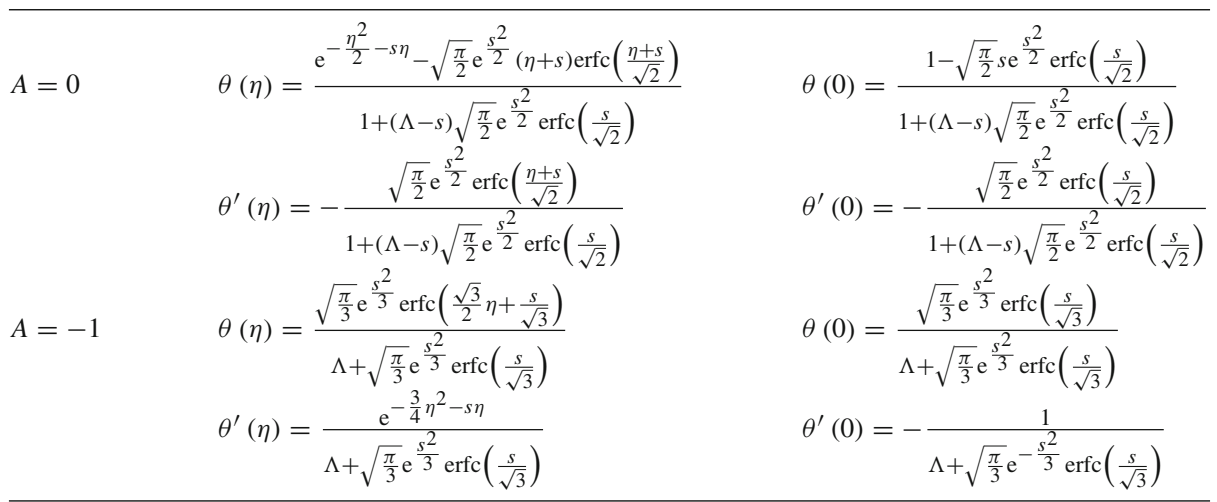

In the impermeable case $s=0$, we have $z_{0}=0, U\left(\frac{1}{2}-a, \frac{1}{2}, 0\right)=\sqrt{\pi} / \Gamma(1-a)$, and the complicated expression of $\Omega$ given by Eq. 16 reduces to

$$
\Omega=\frac{\sqrt{2+|A|} \Gamma(1-a)}{\Gamma\left(\frac{1}{2}-a\right)} \quad(s=0)
$$

In this way, in the general solution (20), we recover for $s=0$ the solution given by Eqs. 18 and 19 of Rohni et al. (2011).

\subsection{The Range $|A|>1$}

A simple inspection of the asymptotic formula (12) emphasizes at a first glance the main message of the present article. Namely, it shows that for $|A|>1$ the exponent $2(1-|A|) /(2+|A|)$ of $\eta$ is negative and thus the asymptotic condition $\theta(\infty)=0$, is satisfied automatically, regardless of the values of $C_{1}$ and $C_{2}$. In other words, in contrast to the case $|A| \leq 1$ where Eq. 13 results as a necessary requirement of the asymptotic condition $\theta(\infty)=0$, in the range $|A|>1$, the condition (13) must not necessarily be imposed. Consequently, for $|A|>1$, only the first boundary condition (4) is effective, yielding with Eqs. 9 and 10 a linear relationship between $C_{1}$ and $C_{2}$, but a second effective relationship does not necessarily exist in this case. Thus, one of the integration constants remains undetermined or, equivalently, either the value of the wall temperature $\theta(0)$ or of the wall temperature gradient $\theta^{\prime}(0)$ can be fixed arbitrarily. Accordingly, the solution (18) which is unique in the range $|A| \leq 1$, does bifurcate for $|A|>1$ in a one-parameter family of infinite many solutions. This means that above the critical value $|A|_{\text {crit }}=1$ of the unsteadiness parameter, the definiteness goes spontaneously broken, and the forced convection problem (3), (4) becomes ill-posed. Obviously, the solution (18) corresponding to Eq. 13 does formally exist also in the range $|A|>1$ (being one of the members of the mentioned family of non-denumerable infinity of solutions), but in this case Eq. 13 becomes an ad-hoc relationship and not a necessary requirement of the asymptotic condition $\theta(\infty)=0$.

To be more specific, we would like to illustrate the arbitrariness described above by an example corresponding to a special value of $|A|$ in the range $|A|>1$. This example is 
suggested by the fact that the basic Eq. 3 can be transcribed in the form:

$$
\left[\theta^{\prime}+\left(\frac{2+|A|}{2} \eta+s\right) \theta\right]^{\prime}-\frac{4-|A|}{2} \theta=0
$$

Now we see that $|A|=4$ is that special value of $A$ for which Eq. 3 admits a first integral which reads

$$
\theta^{\prime}+(3 \eta+s) \theta=K_{1}
$$

where $K_{1}$ is a constant. In the present context, the key feature of Eq. 23 is that the constant $K_{1}$ is necessarily zero only for a solution $\theta(\eta)$ which approaches zero faster than $1 / \eta$ as $\eta \rightarrow \infty$. When, however, $\theta$ decays as $1 / \eta$, the constant $K_{1}$ must necessarily be non-vanishing. This conclusion is in full agreement with the asymptotic formula (12) which shows that for $|A|=4$, the solution decays precisely as $1 / \eta$. In this case, Eq. 23 is non-homogeneous, and thus its general solution is obtained as $\theta(\eta)=\theta_{0}(\eta)+\theta_{p}(\eta)$ where $\theta_{0}(\eta)$ is the general solution:

$$
\theta_{0}(\eta)=K_{2} \mathrm{e}^{-\frac{(3 \eta+s)^{2}}{6}}
$$

of the homogeneous equation $\theta^{\prime}+(3 \eta+s) \theta=0$, and $\theta_{p}(\eta)$ a particular solution of the non-homogeneous Eq. 23. It can be shown that

$$
\theta_{p}(\eta)=K_{1} \sqrt{\frac{\pi}{6}} \mathrm{e}^{-\frac{(3 \eta+s)^{2}}{6}} \operatorname{erfi}\left(\frac{3 \eta+s}{\sqrt{6}}\right)
$$

where erfi $(z)$ denotes the imaginary error function erfi $(x)=-i$ erf $(i x)$ which in turn can be expressed in terms of the famous Dawson integral:

$$
F(x)=\mathrm{e}^{-x^{2}} \int_{0}^{x} \mathrm{e}^{t^{2}} \mathrm{~d} t
$$

as erfi $(x)=2 \pi^{-1 / 2} \mathrm{e}^{x^{2}} F(x)$ (see, e.g., Wolfram 2011). Thus, the general solution of Eq. 23 becomes

$$
\theta(\eta)=K_{2} \mathrm{e}^{-\frac{(3 \eta+s)^{2}}{6}}+K_{1} \sqrt{\frac{2}{3}} F\left(\frac{3 \eta+s}{\sqrt{6}}\right)
$$

For the wall temperature $\theta(0)$ and the wall temperature gradient $\theta^{\prime}(0)$ Eq. 27 gives

$$
\theta(0)=K_{2} \mathrm{e}^{-\frac{s^{2}}{6}}+K_{1} \sqrt{\frac{2}{3}} F\left(\frac{s}{\sqrt{6}}\right), \quad \theta^{\prime}(0)=-s K_{2} \mathrm{e}^{-\frac{s^{2}}{6}}+K_{1}\left[1-\sqrt{\frac{2}{3}} s F\left(\frac{s}{\sqrt{6}}\right)\right]
$$

Substituting Eq. 28 in the first boundary condition (4) we obtain for $K_{1}$ and $K_{2}$ the equation:

$$
\left[\sqrt{\frac{2}{3}}(1+s \Lambda) F\left(\frac{s}{\sqrt{6}}\right)-\Lambda\right] K_{1}+(1+s \Lambda) \mathrm{e}^{-\frac{s^{2}}{6}} K_{2}=1
$$

The second equation necessary to determine the integration constants $K_{1}$ and $K_{2}$ should emerge from the second boundary condition (4). However, bearing in mind that (Wolfram 2011)

$$
F(x) \sim \frac{1}{2 x}+\frac{1}{4 x^{3}}+\cdots \text { for } x \gg 1
$$


Eq. 27 yields

$$
\theta(\eta) \underset{\eta \rightarrow \infty}{\sim} K_{2} \mathrm{e}^{-\frac{3}{2} \eta^{2}}+\frac{K_{1}}{3 \eta} \rightarrow 0
$$

which shows that (i) the condition $\theta(\infty)=0$ is satisfied automatically, and (ii) the asymptotic behavior of $\theta(\eta)$ is dominated by the slowly decaying particular solution (25), in full agreement with the general considerations based on Eq. 12. Therefore, the asymptotic condition $\theta(\infty)=0$ does not furnish a second equation which, together with Eq. 29, would be necessary to determine the constants of integration $K_{1}$ and $K_{2}$ uniquely. As a consequence, for $|A|=4$, Eq. 27 with $K_{1}$ and $K_{2}$ related to each other by Eq. 29, yields a one-parameter family of solutions. For example, in the impermeable case $s=0$, Eq. 29 reduces to $-\Lambda K_{1}+K_{2}=1$ and Eq. 27 results in

$$
\theta(\eta)=\left(1+\Lambda K_{1}\right) \mathrm{e}^{-\frac{3}{2} \eta^{2}}+K_{1} \sqrt{\frac{2}{3}} F\left(\frac{3 \eta}{\sqrt{6}}\right)
$$

which in turn yields $\theta(0)=1+\Lambda K_{1}$ and $\theta^{\prime}(0)=K_{1}$, were $K_{1}$ is still arbitrary. Taking, e.g., $K_{1}=0$, we obtain $\theta(0)=1$ and $\theta^{\prime}(0)=0$, and recover in (32) the solution $\theta(\eta)=\exp \left(-3 \eta^{2} / 2\right)$ given by Rohni et al. (2011). However, $K_{1}=0$ is an ad-hoc choice of $K_{1}$, one among of an infinity of other allowed ones.

\section{Point Mechanical Analogy to the Forced Convection Problem}

In this section, the anomalous bifurcation of the unique solution (18) into a continuum of a non-denumerable infinity of solutions will be illustrated intuitively with the aid of a familiar point mechanical analogy. To this end, we first transcribe Eq. 3 in the form:

$$
\theta^{\prime \prime}=-\gamma \theta^{\prime}-\frac{\partial W}{\partial \theta} \quad(\lambda=0)
$$

where

$$
\gamma=s+\left(1+\frac{|A|}{2}\right) \eta, \quad W=\frac{|A|-1}{2} \theta^{2}
$$

Now we see that the boundary value problem (3), (4) can be reinterpreted as the point mechanical problem of a one-dimensional motion. In this sense, Eq. 33 describes the dissipative motion of a particle of coordinate $\theta$, mass $m=1$, and potential energy $W$ in the presence of a viscous friction force $-\gamma \theta^{\prime}$. The similarity variable $\eta$ of the flow plays the role of time variable of the particle motion. Thus, the friction coefficient is not a constant but a linearly increasing function of time. In Fig. 1, the shape of the potential energy is shown in the ranges $|A| \leq 1$ and $|A|>1$ of the unsteadiness parameter $A$. This potential energy is due to the work of the linear force $-\partial W / \partial \theta=(1-|A|) \theta$ which attracts the particle toward the minimum of $W$ at $\theta=0$ (stable equilibrium point) when $|A|>1$ (red curve), and repels it from the maximum of $W$ at $\theta=0$ (unstable equilibrium point) when $|A| \leq 1$ (blue curve). In the case $|A|=1$, this linear force is absent, $W \equiv 0$, and thus the asymptotic state $\theta=0$ is neutrally (marginally) stable. The boundary conditions (4) require the determination of the initial position $\theta(0)$ and of the initial velocity $\theta^{\prime}(0)$ of the particle, so that (i) between these initial values, still the linear relationship $\theta(0)-\Lambda \theta^{\prime}(0)=1$ holds, and (ii) the particle ends its motion in the origin $\theta=0$ with a vanishing terminal velocity $\theta^{\prime}=0$ as $\eta \rightarrow \infty$ (smoothness condition). The integral energy balance of the motion is given by 


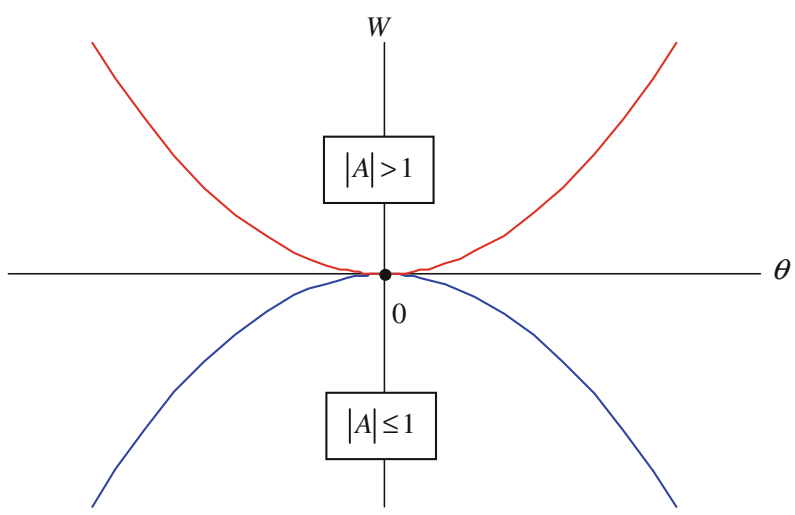

Fig. 1 Potential energy of the analogous particle motion corresponding to the forced convection $(\lambda=0)$ heat transfer problem. The asymptotic state $\theta(\infty)=0$ is a stable equilibrium point of the particle when $|A|>1$ (red curve), and an unstable one when $|A| \leq 1$ (blue curve)

$$
\frac{1}{2}\left[\theta^{\prime}(0)\right]^{2}+W_{0}=\int_{0}^{\infty} \gamma(\eta) \theta^{\prime^{2}}(\eta) d \eta
$$

where $\left[\theta^{\prime}(0)\right]^{2} / 2$ is the initial kinetic energy, and $W_{0}=-(1-|A|)[\theta(0)]^{2} / 2$ is the initial potential energy of the particle. The integral term of Eq. 35 gives the work of the friction force during the particle motion from $\eta=0$ to $\eta=\infty$.

When $|A| \leq 1$, the initial kinetic energy $\left[\theta^{\prime}(0)\right]^{2} / 2$ of the particle is consumed to overcome the friction force, as well as the repulsion force $(1-|A|) \theta$. The latter work enhances the potential energy from $-(1-|A|)[\theta(0)]^{2} / 2<0$ to zero until the particle ends its motion at the maximum of $W$ with a vanishing terminal velocity $\theta^{\prime}(\infty)=0$. For every specified initial position $\theta(0)$ and a given value of $\Lambda$, there exists in this case a unique initial velocity $\theta^{\prime}(0)$, so that the requirements of the boundary conditions (4) (as well as the smoothness requirement $\theta^{\prime}(\infty)=0$ ) are satisfied. This well-defined particle motion toward the maximum of the potential energy $W$ is described by the unique solution (18).

When, on the other hand, $|A|>1$, the dynamics of the particle motion undergoes a dramatic change. In this case, the particle experiences the attraction force $-(|A|-1) \theta$ which increases proportional to the distance $\theta$ from the stable equilibrium point $\theta=0$ corresponding to the minimum of the potential energy $W$ (of the red curve of Fig. 1) and represents an elastic force with the spring constant $k \equiv|A|-1>0$. Accordingly, the particle motion always continues until its whole initial energy, i.e., the sum of its kinetic energy $\left[\theta^{\prime}(0)\right]^{2} / 2$ and potential (elastic) energy $W_{0}=(|A|-1)[\theta(0)]^{2} / 2>0$, is consumed by the work of the friction force $-\gamma \theta^{\prime}$. As a result, the particle comes definitely to rest at the stable equilibrium point $\theta=0$ as $\eta \rightarrow \infty$. The essential difference compared to the previous case $|A| \leq 1$ is that now the particle motion always ends at the stable equilibrium point $\theta=0$, for any choice of the initial data $\theta(0)$ and $\theta^{\prime}(0)$. Therefore, the asymptotic condition, $\theta(\infty)=0$ is always satisfied automatically, no matter where and with which velocity the particle motion has initially been started. In other words, Eq. 3 with the ineffective condition $\theta(\infty)=0$ admits a twoparameter family of solutions which then is restricted by the first boundary condition $\theta(0)=1+\Lambda \theta^{\prime}(0)$ to a one-parameter family, but to a still non-denumerable infinity 
of solutions associated with indefinite values of the reduced Nusselt number $-\theta^{\prime}(0)$, so that

$$
-\infty<\theta^{\prime}(0)<\infty
$$

This is the spontaneous loss of the definiteness above of the critical value $|A|_{\text {crit }}=1$ of the unsteadiness parameter referred to in the title of the present article.

\section{The Mixed Convection Problem}

In this section, we extend the above point mechanical analogy to the mixed convection problem $(\lambda \neq 0)$ and prove that the anomalous bifurcation into a continuum of a non-denumerable infinity of solutions is still present also in this case and does occur above the same critical value $|A|_{\text {crit }}=1$ of the unsteadiness parameter.

As a first step, we substitute $f^{\prime}$ given by the first Eq. 2 into the second one. The resulting equation can then be transcribed in the form:

$$
\theta^{\prime \prime}=-\gamma \theta^{\prime}-\frac{\partial W}{\partial \theta} \quad(\lambda \neq 0)
$$

which coincides formally with the forced convection Eq. 33; however, in the present case we have

$$
\gamma=f(\eta)+\frac{|A|}{2} \eta, \quad W=\frac{|A|-1}{2} \theta^{2}-\frac{\lambda}{3} \theta^{3}
$$

Obviously, in the forced convection case $(\lambda=0)$ where $f(\eta)=s+\eta$, Eq. 38 reduce to Eq. 34 . Bearing in mind that the occurrence of the anomalous bifurcation is a direct consequence of the stability change of the asymptotic state $\theta(\infty)$, in this respect, the difference between the friction coefficients $\gamma$ given by Eqs. 34 and 38 is not decisive. The simple reason is that for large $\eta$ both these expressions approach the same form $\gamma=(1+|A| / 2) \eta$ which implies that the friction coefficient goes to infinity as $\eta \rightarrow \infty$. The essential difference occurs in the expressions of both the potential energies given by Eqs. 34 and 38. This can immediately be recognized by comparing Fig. 1 to Fig. 2. In this respect, let us discuss the two cases $|A| \leq 1$ and $|A|>1$ separately.

\subsection{The Range $|A| \leq 1$}

The range $|A| \leq 1$ of the unsteadiness parameter $A$ has been investigated numerically by Rohni et al. (2011) in some detail, and above of certain critical values $\lambda_{c}$ of the mixed convection parameter $\lambda$, dual temperature solutions have been found (see Figs. 2, 3, 4, 5 of Rohni et al. 2011). This is the main difference compared to the forced convection case $(\lambda=0)$ where the temperature solution (18) is unique. Our aim at this point is to explain the features of the dual mixed convection temperature solutions $\theta(\eta)$ found in Rohni et al. (2011) in terms of the analogous point mechanical motions. To be specific, we consider the example $(A=-1, \lambda=0.5, s=0.5, \Lambda=0.1)$. In this case, the initial positions $\theta(0)$ and velocities $\theta^{\prime}(0)$ of the particle motions corresponding to the dual temperature solutions are $\left(\theta(0)=0.8697, \theta^{\prime}(0)=-1.3030\right)$, and $\left(\theta(0)=0.3534, \theta^{\prime}(0)=-6.4656\right)$, and the respective dual temperature solutions are plotted in Fig. 3. Bearing in mind that in this case the potential energy of the particle is represented by the green curve of Fig. 2, we see that the first one of the dual solutions, which is given by the upper curve of Fig. 3, describes the unidirectional motion of the particle from the initial position $\theta(0)=0.8697$ to the final 


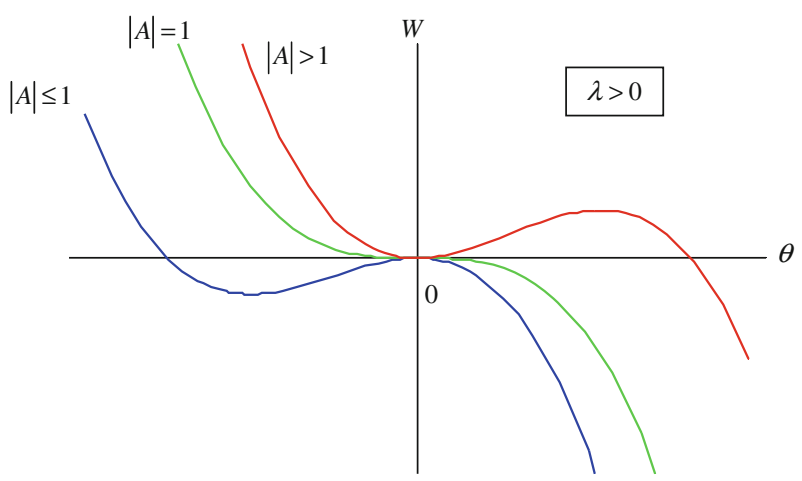

Fig. 2 Potential energy $W$ of the analogous particle motion corresponding to the mixed convection heat transfer problem (with $\lambda>0$ ). The asymptotic state $\theta(\infty)=0$ is a locally stable equilibrium point of the particle when $|A|>1$ (red curve), and a locally unstable one when $|A| \leq 1$ (blue curve). The minimum or maximum of $W$ corresponding to $\theta \neq 0$ is given by $W_{\min / \max }=-(1-|A|)^{3} /\left(6 \lambda^{2}\right)$ and is reached at $\theta_{\min } / \max =-(1-|A|) / \lambda$

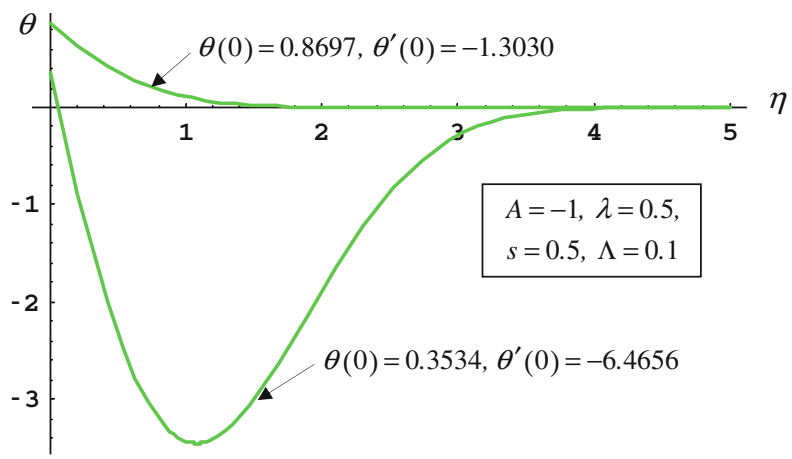

Fig. 3 Dual temperature solutions associated with the indicated values of the parameters $A, \lambda, \Lambda$, and $s$. The corresponding "initial conditions" of the analogous particle motions are also indicated

asymptotic state $\theta(\infty)=0$ (which now is the inflexion point of $W$ ). The dual counterpart of this solution, however, which is given by the lower curve of Fig. 3, describes a bidirectional motion of the particle. Starting from the initial position $\theta(0)=0.3534$, the particle moves to the left, passes the inflexion point $\theta=0$ of $W$ with nonzero velocity, and continues its motion toward a turning point where $\theta^{\prime}=0$ (the minimum of the lower curve of Fig. 3). Here, the particle starts to move in the opposite direction and reaches the inflexion point $\theta=0$ smoothly with vanishing terminal velocity as $\eta \rightarrow \infty$. Therefore, one of the dual temperature solutions found in Rohni et al. (2011) corresponds to a smooth unidirectional motion of the particle from an initial position toward the asymptotic state $\theta(\infty)=0$ (following the upper curve of Fig. 3), and the other one to a bidirectional motion, first overshooting the inflexion point $\theta=0$ of $W$ and then returning to it smoothly as $\eta \rightarrow \infty$ (following the lower curve of Fig. 3). Obviously, this behavior holds for the whole range $-1 \leq A \leq 0$ of the unsteadiness parameter $A$ (the blue and green potential energy curves of Fig. 2). 


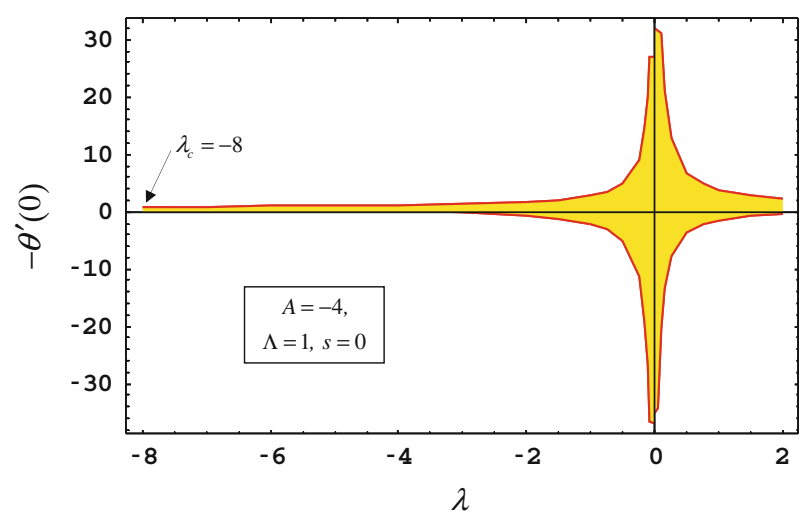

Fig. 4 Dependence of the reduced Nusselt number $-\theta^{\prime}(0)$ on the mixed convection parameter $\lambda$ for the indicated values of $A, \Lambda$, and $s$. The red curves are the border lines (lower and upper bounds) of the domain of existence of temperature solutions $\theta(\eta)$ which is highlighted by yellow. In particular, the whole vertical axis $(\lambda=0)$ is densely filled by the forced convection solutions (32) corresponding to $\theta^{\prime}(0)=K_{1}$, where $K_{1}$ is an arbitrary constant

\subsection{The Range $|A|>1$}

Similar to the forced convection case, in this range of the unsteadiness parameter, the asymptotic state $\theta(\infty)=0$ of the mixed convection temperature field becomes a minimum of the potential energy $W$, i.e., a stable equilibrium point of the particle motion (see the red curve of Fig. 2). The difference arises because, while in the forced convection case the potential well (the red curve of Fig. 1) is infinite at both of its sides and thus the equilibrium point $\theta=0$ is absolutely stable, in the mixed convection cases as an effect of the cubic term $-\lambda \theta^{3} / 3$, the potential barrier becomes finite at one side (the right side and the left side when $\lambda>0$ and $\lambda<0$, respectively) and thus the equilibrium point $\theta=0$ becomes locally stable. The important consequence of this topological feature is that (in the case $A<-1, \lambda \neq 0$ ), for the allowed initial velocities $\theta^{\prime}(0)$ of the particle motion, some finite lower and an upper bound must exist, i.e.,

$$
\theta_{\min }^{\prime}(0) \leq \theta^{\prime}(0) \leq \theta_{\max }^{\prime}(0) .
$$

Otherwise, the particle would leave the potential well at its side of finite height, and thus $\theta$ would approach infinity instead of zero as $\eta \rightarrow \infty$. In other words, similar to the forced convection case, the anomalous bifurcation into a continuum of a non-denumerable infinity of solutions still occurs also in the mixed convection problem above of the same critical value $|A|_{\text {crit }}=1$ of the unsteadiness parameter. The only difference is that, for $|A|>|A|_{\text {crit }}=1$, the arbitrariness of the reduced Nusselt number $-\theta^{\prime}(0)$ is restricted in the mixed convection case to some finite range (39), instead of the doubly infinite domain (36) found in the forced convection problem. The values of the lower and upper bounds of the interval (39) depend for any specified $|A|>1$ on the values of the other three parameters $\lambda, \Lambda$, and $s$ of the problem. This feature is illustrated in Fig. 4 where $-\theta^{\prime}(0)$ has been plotted as a function of the mixed convection parameter $\lambda$ for $A=-4, \Lambda=1$, and $s=0$. The red curves are the border lines (lower and upper bounds) of the domain of existence of the solutions, which extends to the range $\lambda \geq \lambda_{c}=-8$ of the mixed convection parameter. This domain (highlighted by yellow) is densely filled by solutions, i.e., to each of its points, there corresponds a solution of the mixed convection problem (associated with the indicated values of the governing 


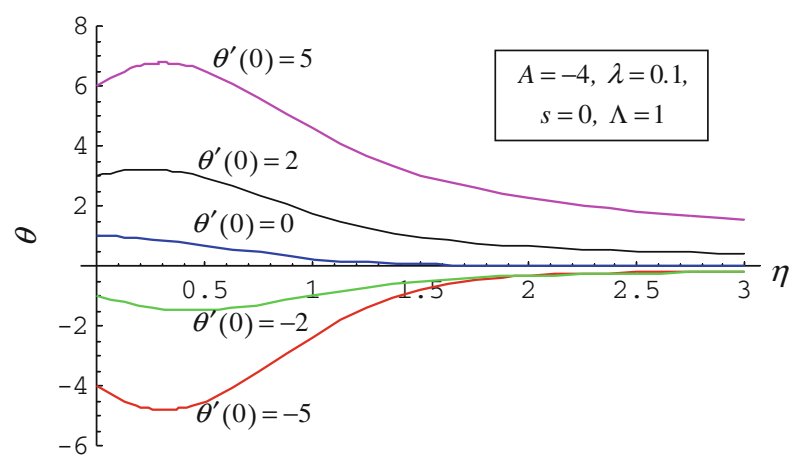

Fig. 5 Five of the continuous infinity of solutions $\theta(\eta)$ filling in Fig. 4 the range $\theta_{\min }^{\prime}(0)=-31.07 \leq$ $\theta^{\prime}(0) \leq \theta_{\max }^{\prime}(0)=20.40$ of the existence domain corresponding to the value $\lambda=0.1$ of the mixed convection parameter

parameters). This feature also holds for the whole vertical axis $(\lambda=0)$ of Fig. 4 which is densely filled by the forced convection solutions (32) corresponding to $\theta^{\prime}(0)=K_{1}$, where $K_{1}$ is an arbitrary constant. To be more specific, in Fig. 5, five of the continuous infinity of solutions filling in Fig. 4 the range (39) at $\lambda=0.1$ have been potted. The lower and upper bounds of the existence domain in this case are $\theta_{\min }^{\prime}(0)=-31.07$ and $\theta_{\max }^{\prime}(0)=20.40$, respectively.

\section{Summary and Conclusions}

The main result of the present article can be substantiated and summarized as follows.

The asymptotic temperature state $\theta(\infty)=0$ can be interpreted as being an equilibrium point of a dissipative dynamical system. In the range of the governing parameters where this equilibrium point is stable (which is the case for $|A|>|A|_{\text {crit }}=1$ ) the boundary condition $\theta(\infty)=0$ is automatically satisfied, regardless of the "initial values" $\theta(0), \theta^{\prime}(0)$, and $f(0)$. This means that the condition $\theta(\infty)=0$ becomes ineffective, and thus the differential problem (2) as a third-order boundary value problem with only two effective boundary conditions becomes automatically underspecified (ill-posed). As a consequence, in the range $|A|>|A|_{\text {crit }}=1$, a continuum of a non-denumerable infinity of solutions occurs and, accordingly, the reduced Nusselt number $-\theta^{\prime}(0)$ becomes indeterminate. It can take any value between $-\infty$ and $+\infty$ in the forced convection case (where the equilibrium point $\theta=0$ is absolutely stable) and in a finite range $\theta_{\min }^{\prime}(0) \leq \theta^{\prime}(0) \leq \theta_{\max }^{\prime}(0)$ in the mixed convection case (where the equilibrium point $\theta=0$ is locally stable).

It is worth mentioning here that the anomalous bifurcation of a well-defined solution into a continuum of solutions has also been observed in the classical Cheng-Minkowycz forced and free convection problems (see Magyari and Aly 2006; Liao and Magyari 2006, respectively). In these cases, the parameter governing this phenomenon is the exponent $\lambda$ of the power-law wall temperature distribution $T_{\mathrm{w}}-T_{\infty} \sim x^{\lambda}$, and the mentioned loss of definiteness occurs for negative values of $\lambda$, i.e., for $|\lambda|>|\lambda|_{\text {crit }}=0$ (see Fig. 1 of Liao and Magyari 2006). Moreover, the described loss of definiteness can be encountered not only in the porous media, but also in the Falkner-Skan flow and heat transfer problems of clear viscous fluids. This spontaneous loss of the definiteness has a topological origin which resides in the structure 
of the governing equations, and thus, it is unavoidable. Actually, it must be interpreted as a further insufficiency of the boundary layer approximation.

\section{References}

Abramowitz, M., Stegun, I.S.: Handbook of Mathematical Functions. Dover Publishers, New York (1965)

Liao, S.-J., Magyari, E.: Exponentially decaying boundary layers as limiting cases of families of algebraically decaying ones. J. Appl. Math. Phys. (ZAMP) 57, 777-792 (2006)

Magyari, E., Aly, E.H.: Exact analytical solution for a thermal boundary-layer flow in a saturated porous medium. Appl. Math. Lett. 19, 1351-1355 (2006)

Mukhopadhyay, S., Andersson, H.: Effects of slip and hear transfer analysis of flow over an unsteady stretching surface. Heat Mass Transf. 45, 1447-1452 (2009)

Rohni, A.M, Ahmad, S., Pop, I., Merkin, J.H.: Unsteady mixed convection boundary layer with suction and temperature slip effects near to stagnation-point on a vertical permeable surface embedded in a porous medium. Transp. Porous Media. Online 14 Oct 2011

Wolfram MathWorld. http://mathworld.wolfram.com/DawsonsIntegral.html (2011)

Yang, K.T.: Unsteady laminar boundary layers in an incompressible stagnation flow. Trans. ASME. J. Appl. Mech. 25, 421-427 (1958) 